\title{
Inactivation of Wnt-LRP5 signaling suppresses the proliferation and migration of ovarian cancer cells
}

\author{
Jing Hong, Zeyu Xie, Zhihua Yang, Fangyao Yang, Hai Liao, Shuquan Rao, Xinhe Huang^ \\ School of Life Science and Engineering, Southwest Jiaotong University, Chengdu, China \\ Contributions: (I) Conception and design: S Rao, H Liao; (II) Administrative support: X Huang; (III) Provision of study materials or patients: J Hong, \\ Z Xie, Z Yang, F Yang; (IV) Collection and assembly of data: J Hong, Z Xie, Z Yang; (V) Data analysis and interpretation: J Hong, Z Xie; (VI) \\ Manuscript writing: All authors; (VII) Final approval of manuscript: All authors. \\ Correspondence to: Xinhe Huang. School of Life Science and Engineering, Southwest Jiaotong University, No. 111, North 1st section, 2nd Ring Road, \\ Chengdu 610031, China. Email: xinhehuang@swjtu.edu.cn.
}

\begin{abstract}
Background: Ovarian cancer $(\mathrm{OCa})$ is the most lethal gynecological malignant tumor, with few or no specific symptoms in its early stage. There are many signaling pathways involved in the process of OCa progression, among which the highly complex Wnt signaling pathway plays a unique role in the occurrence and development of $\mathrm{OCa}$ because of its functions of regulating gene expression, cell proliferation, migration, and invasion. Lipoprotein associated receptor protein 5/6 (LRP5/6) binds to activate this key pathway. Therefore, it is very important to study the mechanism of Wnt-LRP5 signaling pathway in the proliferation and migration of OCa.
\end{abstract}

Methods: In the present study, we have investigated the role of Wnt-LRP5 signaling pathway in OCa proliferation and migration for the first time using the dominant negative plasmid of LRP5 (DN-LRP5) and human OCa cells HO8910PM plus in a mouse model.

Results: Our data showed inactivation of LRP5 resulted in shift of epithelial-mesenchymal transition (EMT), rearrangement of the cytoskeleton, lowered activity of pro-proliferation and pro-migration cancer signaling pathways including Akt, p38 and NF- $\mathrm{B}$, eventually decreased proliferation and migration of OCa cells HO8910PM in vitro. Moreover, in vivo OCa-DN-LRP5 mouse model developed significantly smaller tumors as determined by inoculation of HO8910PM-DN-LRP5 cells into nude mice.

Conclusions: Collectively, our results demonstrate the dominant role of Wnt-LRP5 in OCa proliferation and migration and its potential as a valuable therapeutic target.

Keywords: Wnt; lipoprotein associated receptor protein 5 (LRP5); ovarian cancer (OCa); proliferation; migration

Submitted Dec 18, 2020. Accepted for publication Mar 05, 2021.

doi: $10.21037 /$ tcr-20-3462

View this article at: http://dx.doi.org/10.21037/tcr-20-3462

\section{Introduction}

Ovarian cancer (OCa) is one of the most common female malignant tumors with high mortality. It is the second most common female malignant tumor in terms of mortality (1). In the past 30 years, the 5 -year survival rate of OCa patients is less than $30 \%$. A major contributor to the high mortality rate is the fact that $70 \%$ of women with OCa initially manifests with little or no specific symptoms at the early stage. Therefore, patients present with metastases and invasion throughout the peritoneal cavity (2). Due to lack of effective early detection methods, OCa is often diagnosed as advanced disease clinically. While the mortality of OCa remains highest, the etiology of OCa is still elusive.

Conserved Wnt signaling is generally active during

^ ORCID: 0000-0003-4190-0737. 
human development and growth activities, and plays an important role in physiological activities including embryonic development, organ growth and maintenance of various functions (3-6). Mutations in Wnt genes or components of the Wnt signaling pathway can cause various human diseases, such as developmental defects, and their abnormal functions can also cause cancer (7). In addition, there is growing evidence to show the cross-talk between Wnt signaling pathway and other signaling pathways including Notch (8) and Sonic Hedgehog (9) signaling pathways, which has implications for Wnt signaling pathway in therapeutic interventions of multiple cancers. As currently understood, Wnt proteins bind to receptors of the Frizzled proteins and lipoprotein receptor-related proteins 5/6 (LRP5/6) on the cell surface to further exert its physiological function (10). LRP5 is one member of low density lipoprotein receptor (LDLP) family and is found to be involved in fat metabolism mostly (11). As a co-receptor of the Wnt signaling pathway, LRP5 participates in the development of embryo (12), and especially the development of bone (13) and eye (14). Several line of evidence show the important physiological function of LRP5, for instance, LRP5 triggers osteoporosis-pseudoglioma syndrome (OPPG), LRP5 is positively correlated with the activity of osteosarcoma cells (15), and mutations in LRP5 directly affect bone acquisition and eye development (16), cause insulin-dependent diabetes (17) and non-syndromic hearing loss (18).

Recently, LRP5 was found to be involved in multiple tumors progression though the exact mechanism is unknown. Rabbani et al. showed LRP5 regulated the process of epithelial-mesenchymal transition (EMT) and mediated the proliferation and migration of prostate cancer cells (19). Furthermore, LRP5 has proven to affect or directly interact with new tumor-associated factors such as STK40 (20), JAM3 (21), TG2 (22), Hsp90ab1 (23), in different cancer cells to regulate tumorigenesis and migration. In addition, a variety of natural products and synthetic compounds also exert their anticancer activity by acting on Wnt/LRP5 signaling $(24,25)$, all of which suggests LRP5 could be a new anti-cancer target.

In our research, we have immediate study the role of Wnt-LRP5 signaling pathway in OCa progression by transfection of the dominant negative plasmid of LRP5 (DN-LRP5) into highly metastatic human OCa cells HO8910PM. The effect of transfection of DN-LRP5 plasmid and abrogation of Wnt-LRP5 pathway was examined on OCa cells characteristics in vitro and on mouse tumor growth in vivo. We present the following article in accordance with the ARRIVE reporting checklist (available at http://dx.doi.org/10.21037/tcr-20-3462).

\section{Methods}

\section{Cell culture}

Human liver cell line (L02, Chang Liver), human liver cancer cell lines (HepG2, HepG2.2.1.5, Hep3B, 7721, PLC, Huh7, 97-H, LM3, FOCUS, SNU398, sk-hep-1), human colorectal cancer cell lines (LOVO, SW480, HCT116, SW620), human OCa cells (A2780, HO8910, SK-OV-3, HO8910PM), human lung cancer cell lines (H460, H446), human embryonic kidney cell line (HEK293T) were from the American Type Culture Collection and maintained in Dulbecco's Modified Eagle's Medium (DMEM) with $10 \%$ fetal bovine serum (FBS). HO8910PM was infected with DN-LRP5 lentiviral and selected with puromycin to obtain the stable clone of HO8910PM/DN-LRP5, then maintained in DMEM with 10\% FBS and puromycin for future experiments.

\section{MTT assay}

The logarithmic growth phase HO8910PM and HO8910PM/DN-LRP5 cells were plated to 96-well culture plates with a density of $10^{4}$ cells $/ \mathrm{mL}$ in medium containing $10 \%$ FBS. MTT was added to the cell culture at specific time points with a final concentration of $1 \mathrm{mg} / \mathrm{mL}$ and incubated at $37^{\circ} \mathrm{C}$ for another $3 \mathrm{~h}$. The optical absorbance at $570 \mathrm{~nm}$ was measured to determine the change of cell density (26).

\section{Western blotting analysis}

Protein lysates $(20-80 \mu \mathrm{g})$ were obtained by lying HO8910PM and HO8910PM/DN-LRP5 cells, and then boiled at $100{ }^{\circ} \mathrm{C}$ for $5-10$ minutes and resolved by $10 \%$ SDS-PAGE. Proteins were transferred to PVDF membranes, and sealed with $5 \%$ skim milk, the membranes were treated with specific primary antibodies. Then horseradish peroxidase-conjugated secondary antibodies were used to treat the blots for $1 \mathrm{~h}$. The blots were visualized through enhanced chemiluminescence.

\section{Wound-bealing analysis}

HO8910PM and HO8910PM/DN-LRP5 cells were plated 
to a 6-well culture plates with a density of $10^{6}$ cells $/ \mathrm{mL}$. After adherence, draw a vertical parallel wound on the cell layer using a p10 tip, then the medium was removed and cells were rinsed 3 times with sterile PBS to remove shed cells and cell debris. Proliferation of the cells in DMEM medium containing $2 \%$ FBS were measured to assay the rate of wound healing (27).

\section{Transwell migration assay}

$2 \times 10^{4}$ cells were mixed and added to the inside of the transwell, and the transwell was placed in a 24-well plate containing $500 \mu \mathrm{L}$ of complete medium, and cells were cultured for another 24 hours. Then the transwell was fixed with methanol for $25 \mathrm{~min}$, stained with crystal violet for 45 min and washed with PBS three times, and the cell layer was gently scraped off with a medical cotton swab. Add $200 \mu \mathrm{L}$ of sterile water or PBS to the inside of the transwell after cleaning. Photograph the transwell with an inverted microscope and count the number of cells (28).

\section{Actin staining}

HO8910PM and HO8910PM/DN-LRP5 cells were grown on coverslips for 24 hours, then fixed with Ready-made 4\% paraformaldehyde at room temperature for 10 minutes, diluted Triton X-100 with PBS to a final concentration of $0.1 \%$, and then penetrated the cells 20 minutes, then fix with $5 \%$ bovine serum albumin (BSA) for 1 hour, and stain with FITC-Phalloidin for 2 hours at room temperature and dark conditions. After washing twice with PBS, it was counter-stained with DAPI for $10 \mathrm{~min}$. Finally, the distribution of F-actin (29) was observed by laser confocal microscope.

\section{Animal protocols}

Male BALB/C nude mice were grown to 6 weeks of age for tumor formation experiments. The experiment was divided into two groups. The experiment was performed under a project license [No. SYXK(Chuan)2014-189] granted by institutional ethics board of Southwest Jiaotong University, in compliance with Sichuan guidelines for the care and use of animals. The prepared cell suspensions HO8910PM and HO8910PM/DN-LRP5 $\left(5 \times 10^{7}\right.$ cells/mL $)$ were aspirated under sterile conditions, and $100 \mu \mathrm{L}$ of cell suspension was injected into the right neck of each nude mouse $\left(5 \times 10^{6}\right.$ cells/mouse). Each nude mouse was labeled and kept under sterile conditions. The lengths of the long and short axes of tumors were measured every two days, and the tumor volume was calculated according to the formula $\mathrm{V}=$ $0.5236 \times \mathrm{L}_{1} \times \mathrm{L}_{2} \times \mathrm{L}_{2}$ (where $\mathrm{L}_{1}$ is the long axis of the tumor, and $\mathrm{L}_{2}$ is the short axis of the tumor).

\section{Statistical analysis}

Data were presented as the mean $\pm \mathrm{SD}$. At least three independent experiments were performed. Comparisons of the experimental data were assessed using an independent two-sample $t$-test at $\mathrm{P}<0.05$ level of significance.

\section{Results}

\section{DN-LRP5 suppresses the proliferation of HO8910PM cells}

In the previous study, we found LRP5 had significantly increased expression in highly metastatic colorectal cancer cells SW620 and OCa cells HO8910PM, while little expression in non-metastatic colorectal cancer cell line SW480 and OCa cell line HO8910 through western blot experiments (Appendix 1, Figure S1), which suggests that LRP5 may regulate cell migration and invasion. Then we constructed dominant negative LRP5 in HO8910PM cell line to explore the functions of LRP5 (Appendix 1, Figure S2), as expected, we observed that HO8910PM/DN-LRP5 cells grew much slower than that of HO8910PM. Further growth rate measurements by MTT assay indicated DN-LRP5 significantly inhibited the proliferation of HO8910PM cells (Figure 1).

\section{DN-LRP5 inbibits the migration of HO8910PM}

To explore effects of DN-LRP5 on cell migration, HO8910PM and HO8910PM/DN-LRP5 cell lines were employed to perform the wound healing and the transwell experiments in a time course manner. The results showed the wound healing rate of HO8910PM cells was $93 \%$ at $48 \mathrm{~h}$, which is much higher than a $32 \%$ of wound healing rate at $48 \mathrm{~h}$ for HO8910PM/DN-LRP5 cells (Figure 2A,B), indicating DN-LRP5 can significantly inhibit the migration of HO8910PM cells. Similarly, the transwell assay showed that HO8910PM/DN-LRP5 migrated much lower than HO8910PM, indicating that DN-LRP5 inhibited the migration of HO8910PM cells significantly with an inhibition rate of around $45 \%$ (Figure $2 C, D$ ). 


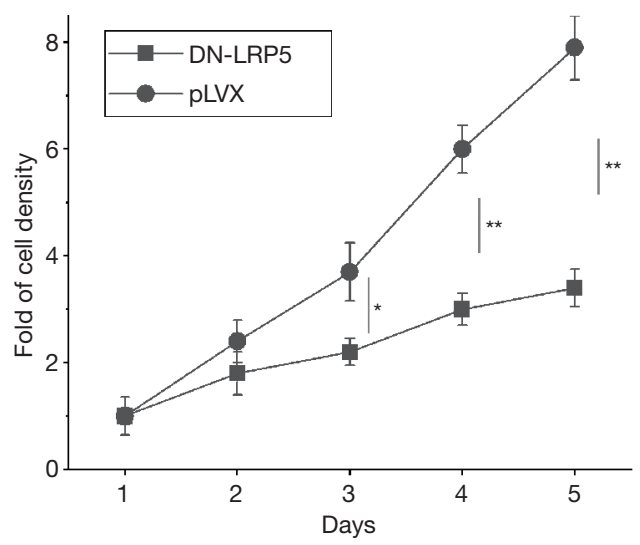

Figure 1 DN-LRP5 suppressed the proliferation of HO8910PM. The proliferation of HO8910PM and HO8910PM/DN-LRP5 was measured by MTT assay. DN-LRP5, dominant negative plasmid of LRP5. ${ }^{*} \mathrm{P}<0.05 ;{ }^{* *} \mathrm{P}<0.01$.

\section{DN-LRP5 rearranges the cytoskeleton of HO8910PM}

The arrangement of the cytoskeleton is closely related to the morphology and motility of the cells (30). Usually, the rearrangement of the cytoskeleton triggers changes in the structure of the microfilaments, resulting in an increase in the production of protrusive structures and an increase in the ability of tumor cells to move and migrate (31). As expected, HO8910PM cells had obvious protrusive structures (Figure 2E, left panel), showing high metastatic characteristics, while HO8910PM/DN-LRP5 showed no obvious protrusive structures, suggesting DN-LRP5 rearranged the cytoskeleton and inhibited the mobility of HO8910PM cells (Figure 2E, right panel).

\section{$D N-L R P 5$ regulates EMT process}

EMT plays key roles in tumor metastasis. EMT molecular markers (for example, E-cadherin, Keratin 8/18, vimentin and fibronectin) are the hallmark events during EMT (32), accordingly, we detected the key molecules of EMT in both HO8910PM and HO8910PM/DN-LRP5 cells by western blotting, seeing the Appendix 1. As shown in Figure $3 A, B$ and Figure S3A,B, HO8910PM/DN-LRP5 cells showed decreased expression of mesenchymal markers vimentin and fibronectin and increased expression of epithelial markers E-cadherin and Keratin 8/18, suggesting DN-LRP5 somehow reversed EMT process of HO8910PM cells and participated the regulation of cell migration.

\section{$D N-L R P 5$ regulates multiple cancer-associated patbways}

Wnt signaling pathway is often implicated in regulation of other cancer signaling pathways, for example, the HGF/ c-Met (33), the PI3 Kinase/Akt (34), the MAPK (35) and $\mathrm{NF}-\mathrm{\kappa B}$ (36) pathways to play a pivot role in tumor formation and metastasis. With this context, we explored if DNLRP5 has any effects on classical cancer signaling pathways including the Akt, p38, HGF/c-Met and NF- $\mathrm{BB}$, seeing the Appendix 1. As shown in Figure $3 C$ and Figure S3C, DN-LRP5 significantly down-regulated the activity of the Akt, p38, HGF/c-Met and NF-кB, while had little effects on the GSK $3 \beta$, the Stat 3 and the Smad3 signaling pathways, suggesting Wnt-LRP5 cross-talked with those signaling pathways by a synergy manner possibly though the detailed mechanisms are elusive.

\section{DN-LRP5 inhibits tumor proliferation of nude mice}

Next, we were concerned about the effectiveness of DNLRP5 on tumor inhibition in nude mice. With this purpose, nude mice were injected with HO8910PM and HO8910PM/DN-LRP5 cells at the same amount of cells respectively. From the fourth day after injection, the tumor volume was recorded every 2 days. The results showed that DN-LRP5 significantly inhibited tumor growth in nude mice in a time-dependent manner, with an inhibition rate high to around $90 \%$ eventually (Figure 4).

\section{Discussion}

The Wnt signaling pathway is super complex, containing 19 different Wnt ligands, 10 Frizzled receptors, several helper receptors, and numerous downstream target proteins, which have been evaluated by researchers for many years (37). Even though, the Wnt signaling pathway can be broadly classified into two categories, the canonical $\mathrm{Wnt} / \beta$-catenin pathway and non-canonical pathways, such as the $W n t / \mathrm{Ca}^{2+}$ pathway, the Wnt/PCP pathway, and the specificity of each pathway depends on different cell types, WNTs, SFRPs, WIF1, DKKs, Frizzled, co-receptors (LRP5/6), and regulation of Wnt signaling regulators in the cytoplasm (38). Except the developmental function of Wnt signaling pathway, recent studies have shown that inhibition of Wnt signaling activity can significantly inhibit the growth of tumor cells such as bladder cancer, rectal cancer, and liver cancer (39) though the detailed mechanisms are elusive. 
A
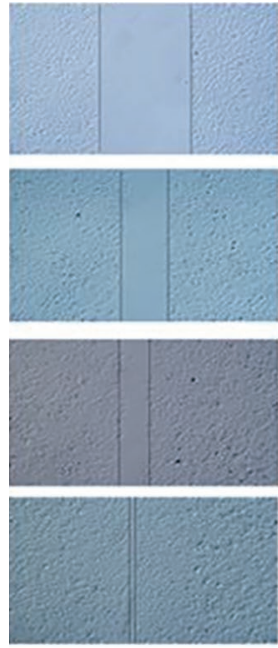

$\mathrm{pLVX}$
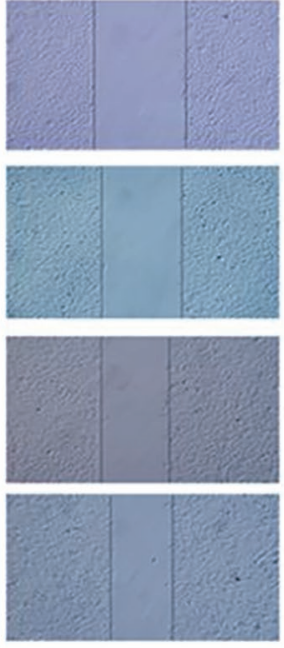

DN-LRP5

C

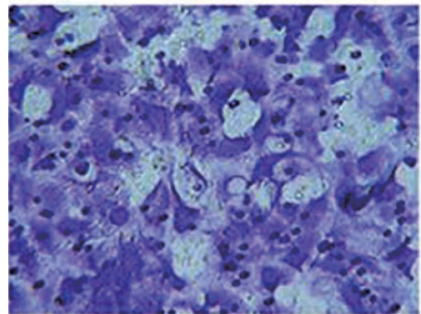

pLVX

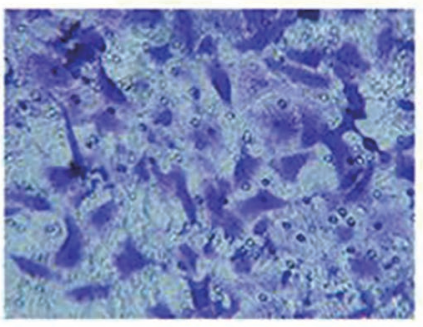

DN-LRP5

E

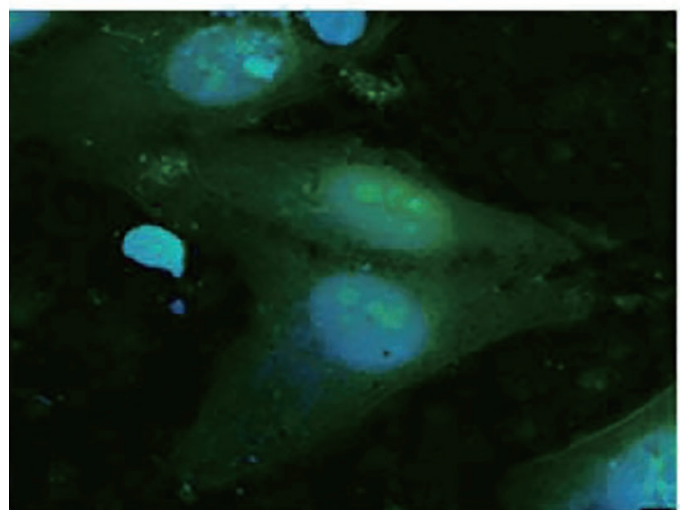

pLVX

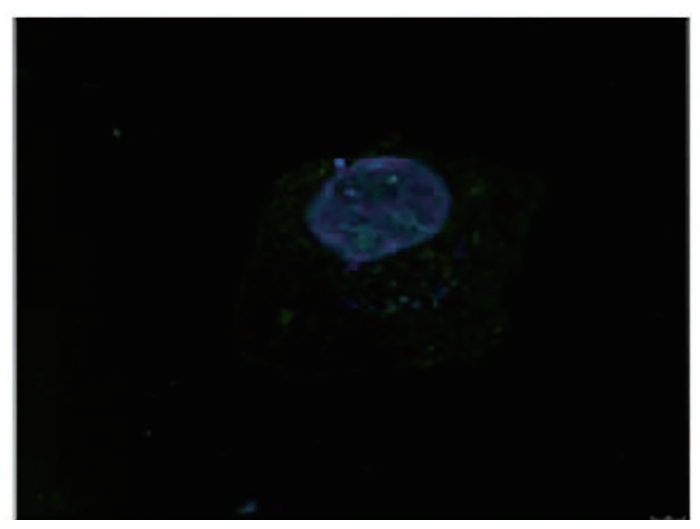

DN-LRP5

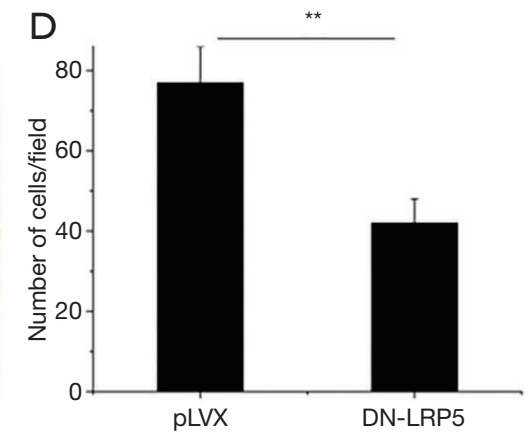

B

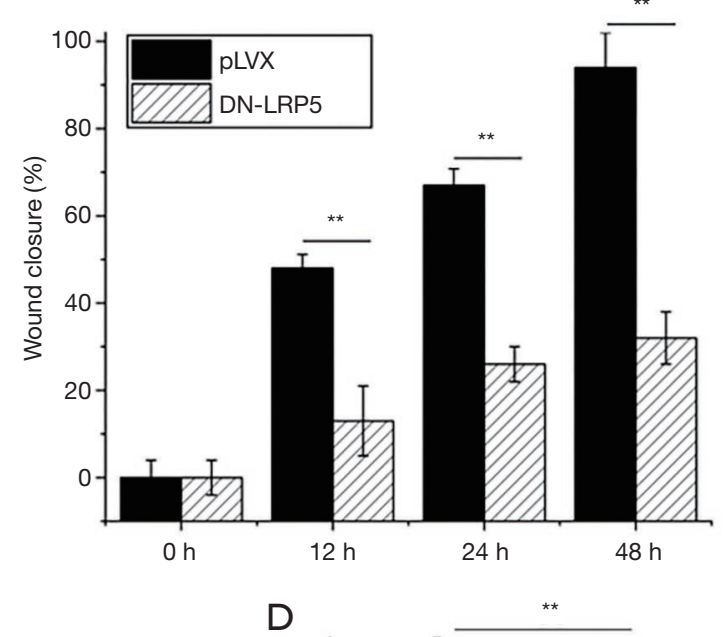

Figure 2 DN-LRP5 inhibited the migration of HO8910PM. (A) Migration of HO8910PM and HO8910PM/DN-LRP5 by straight scratch assay (×40). (B) Wound healing rate of HO8910PM and HO8910PM/DN-LRP5 at 0, 12, 24 and 48 h, respectively. (C) Invasion of HO8910PM and HO8910PM/DN-LRP5 cells in transwell by crystal violet staining ( $\times 200)$. (D) Statistical analysis for invasion of HO8910PM and HO8910PM/DN-LRP5 cells. (E) Cytoskeleton observation of HO8910PM and HO8910PM/DN-LRP5 cells by FITCphalloidin staining assay $(\times 400) .{ }^{* *}, \mathrm{P}<0.01$. DN-LRP5, dominant negative plasmid of LRP5.

Here, we found Wnt-LRP5 is involved in progression of OCa cells, which adds a new possibility for OCa detection and treatment by targeting this classical signaling pathway.

As a co-receptor of Wnt signaling pathway, LRP5 was thought to be involved in the regulation of development (for example, bone and eye development) mostly in previous research. The role of LRP5 in tumors regulation was rarely reported. In terms of the specific role of LRP5 in tumor cells, studies have shown that the dominant negative mutant of LRP5 overexpressing in prostate cancer PC-3 cells can 

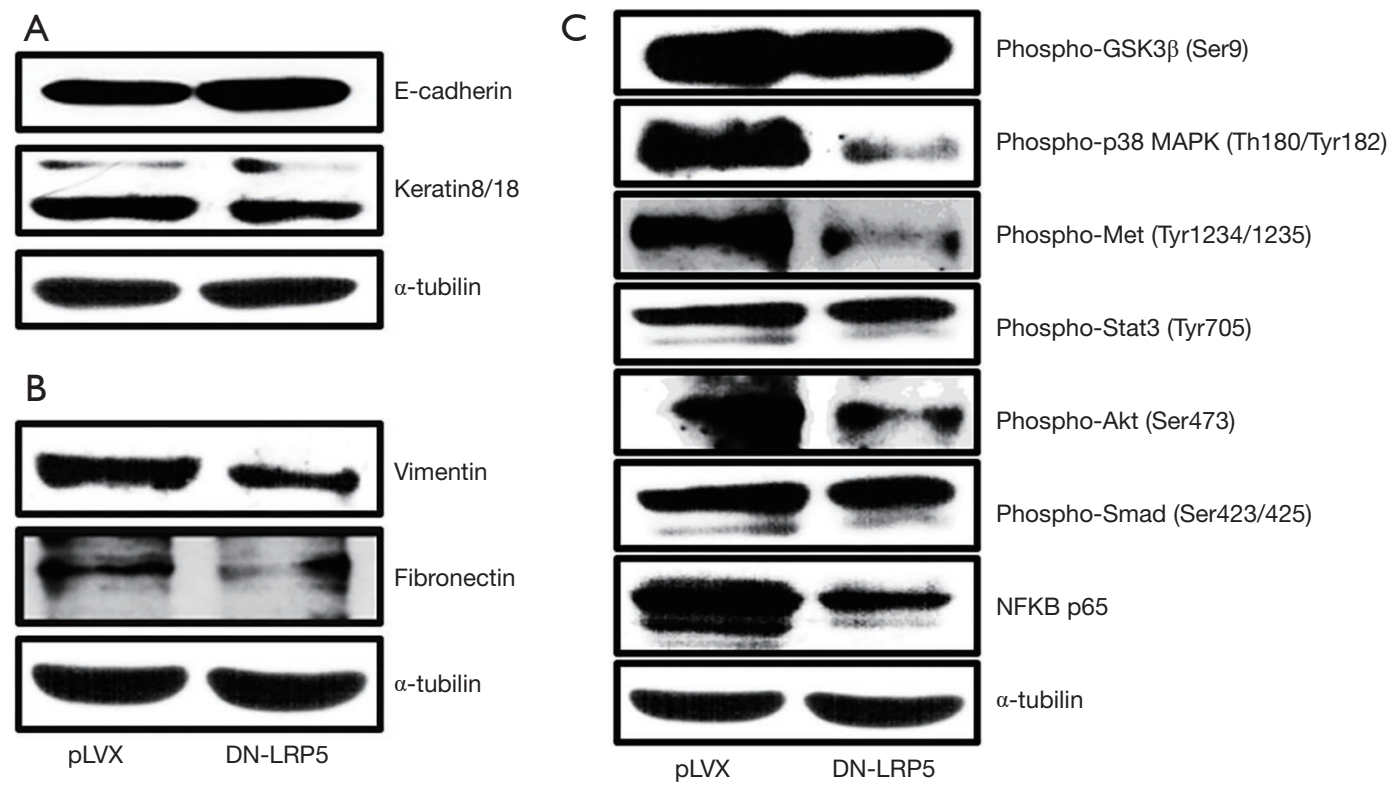

Figure 3 DN-LRP5 regulates EMT and multiple cancer-associated pathways. (A) Epithelial markers detection by western blotting; (B) mesenchymal markers detection by western blotting; (C) multiple cancer signaling pathways detection by western blotting. DN-LRP5, dominant negative plasmid of LRP5; EMT, epithelial-mesenchymal transition.

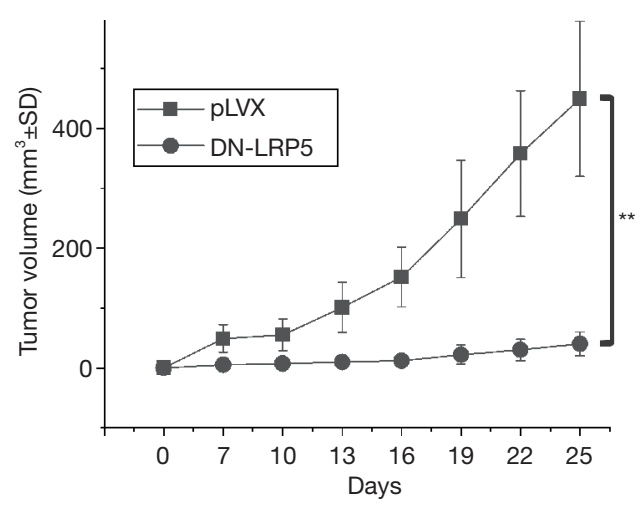

Figure 4 DN-LRP5 inhibits tumor proliferation of nude mice. Nude mice were injected with HO8910PM and HO8910PM/DNLRP5 cells and tumor formation was recorded and measured every 2 days. Tumor volume $=0.5236 \times$ long axis $\times$ short axis $\times 2$. **, $\mathrm{P}<0.01$. DN-LRP5, dominant negative plasmid of LRP5.

inhibit the infiltration and migration of tumor cells and formation of subcutaneous xenograft tumors in nude mice, and the main mechanism is by up-regulating E-cadherin expression and inhibiting MMPs activity (40). After that, overexpression of DN-LRP5 also significantly inhibited the infiltration and migration of osteosarcoma cells HT1080 and SW872, as well as tumorigenicity in nude mice, via down-regulation of hepatocyte growth factor receptor c-Met expression and inhibition of c-Met-mediated activity (41). In our current study, we found DN-LRP5 significantly inhibited the proliferation and migration of OCa cells in vitro (Figure 1) and tumor formation of mouse in vivo (Figure 4), our data plus the results from other group suggests LRP5 could be a new anti-tumor target.

In addition to the migration assay in vitro, the invasive phenotype of OCa may need to change the molecular structure from epithelial cells to mesenchymal cells. Down regulation of $\mathrm{E}$-cadherin and up regulation of $\mathrm{N}$-cadherin are associated with $\mathrm{OCa}$ progression, which is a common phenomenon in EMT sites during normal development and cancer (42). In our study, suppressing LRP5 expression in HO8910PM cells weakened cellular mobility, accompanying an obviously upregulation of epithelial markers E-cadherin and Keratin8/18 (Figure $3 A$ ) as well as downregulation of mesenchymal markers fibronectin and vimentin (Figure 3B), similar to the reversal of EMT (43). In summary, the reversal of EMT and the migration and actin staining assay in vitro suggested that LRP5 affected migration of $\mathrm{OCa}$ cells.

Activated phospho-Akt could promote cell proliferation via exerting anti-apoptotic effects through multiple downstream pathways including mTOR, Bcl-2, Caspase-9, 
NF- $\mathrm{B}$ (34). Similarly, phosphorylation activation of p38 MAPK (35) and c-Met (33) and combination of NF- $\kappa B$ and p65 (36) could activate the corresponding downstream pathway to promote cell proliferation and migration. The western blot results suggested that DN-LRP5 prevented phosphorylation activation of Akt, p38 MAPK and $\mathrm{c}-$ Met and combination of NF- $\mathrm{KB}$ and $\mathrm{p} 65$ to inhibit cell proliferation and migration. This indicates that Wnt/LRP5 can cross talk with HGF/c-Met, Akt, p38 MAPK and NF$\kappa \mathrm{B}$ signaling pathways to regulate cell proliferation and migration.

Our study successfully established a stable expression cell line of DN-LRP5 in OCa cells and proved that DN-LRP5 has a certain inhibitory effect on the growth and migration of OCa cells. This lays a foundation for further exploration of the anti-OCa and molecular mechanisms of DN-LRP5, thus building a framework for the development of antitumor drugs against Wnt signaling pathway.

\section{Acknowledgments}

Funding: This work was supported by Sichuan Province Miaozi Program (No. 2020110), the Key R\&D Projects of Sichuan Province (No. 2018SZ0061) and the Fundamental Research Funds for the Central Universities of China (No. LKPY2020-L376).

\section{Footnote}

Reporting Checklist: The authors have completed the ARRIVE reporting checklist. Available at http://dx.doi. org/10.21037/tcr-20-3462

Data Sharing Statement: Available at http://dx.doi. org/10.21037/tcr-20-3462

Conflicts of Interest: All authors have completed the ICMJE uniform disclosure form (available at http://dx.doi. org/10.21037/tcr-20-3462). The authors have no conflicts of interest to declare.

Ethical Statement: The authors are accountable for all aspects of the work in ensuring that questions related to the accuracy or integrity of any part of the work are appropriately investigated and resolved. Experiments were performed under a project license [No. SYXK(Chuan)2014-189] granted by institutional ethics board of Southwest Jiaotong University, in compliance with
Sichuan guidelines for the care and use of animals.

Open Access Statement: This is an Open Access article distributed in accordance with the Creative Commons Attribution-NonCommercial-NoDerivs 4.0 International License (CC BY-NC-ND 4.0), which permits the noncommercial replication and distribution of the article with the strict proviso that no changes or edits are made and the original work is properly cited (including links to both the formal publication through the relevant DOI and the license). See: https://creativecommons.org/licenses/by-nc-nd/4.0/.

\section{References}

1. Siegel RL, Miller KD, Jemal A. Cancer Statistics, 2017. CA Cancer J Clin 2017;67:7-30.

2. Vaughan S, Coward JI, Bast RC Jr, et al. Rethinking ovarian cancer: recommendations for improving outcomes. Nat Rev Cancer 2011;11:719-25.

3. Brafman D, Willert $\mathrm{K}$. Wnt/beta-catenin signaling during early vertebrate neural development. Dev Neurobiol 2017;77:1239-59.

4. Hussain $\mathrm{M}, \mathrm{Xu} \mathrm{C}, \mathrm{Lu} \mathrm{M}$, et al. Wnt/beta-catenin signaling links embryonic lung development and asthmatic airway remodeling. Biochim Biophys Acta Mol Basis Dis 2017;1863:3226-42.

5. Veltri A, Lang C, Lien WH. Concise Review: Wnt Signaling Pathways in Skin Development and Epidermal Stem Cells. Stem Cells 2018;36:22-35.

6. Girardi F, Le Grand F. Wnt Signaling in Skeletal Muscle Development and Regeneration. Prog Mol Biol Transl Sci 2018;153:157-79.

7. Nusse R, Clevers H. Wnt/beta-Catenin Signaling, Disease, and Emerging Therapeutic Modalities. Cell 2017;169:985-99.

8. Collu GM, Hidalgo-Sastre A, Brennan K. Wnt-Notch signalling crosstalk in development and disease. Cell Mol Life Sci 2014;71:3553-67.

9. Noubissi FK, Yedjou CG, Spiegelman VS, et al. CrossTalk between Wnt and Hh Signaling Pathways in the Pathology of Basal Cell Carcinoma. Int J Environ Res Public Health 2018;15:1442.

10. MacDonald BT, He X. Frizzled and LRP5/6 receptors for Wnt/beta-catenin signaling. Cold Spring Harb Perspect Biol 2012;4:a007880.

11. Go GW, Mani A. Low-density lipoprotein receptor (LDLR) family orchestrates cholesterol homeostasis. Yale J Biol Med 2012;85:19-28. 
12. Chen Q, Zhang Y, Lu J, et al. Embryo-uterine cross-talk during implantation: the role of Wnt signaling. Mol Hum Reprod 2009; 15:215-21.

13. Kato M, Patel MS, Levasseur R, et al. Cbfa1-independent decrease in osteoblast proliferation, osteopenia, and persistent embryonic eye vascularization in mice deficient in Lrp5, a Wnt coreceptor. J Cell Biol 2002;157:303-14.

14. Fujimura N. WNT/beta-Catenin Signaling in Vertebrate Eye Development. Front Cell Dev Biol 2016;4:138.

15. Hoang BH, Kubo T, Healey JH, et al. Dickkopf 3 inhibits invasion and motility of Saos-2 osteosarcoma cells by modulating the Wnt-beta-catenin pathway. Cancer Res 2004;64:2734-9.

16. Gong Y, Slee RB, Fukai N, et al. LDL receptorrelated protein 5 (LRP5) affects bone accrual and eye development. Cell 2001;107:513-23.

17. Ferrari SL, Deutsch S, Choudhury U, et al. Polymorphisms in the low-density lipoprotein receptorrelated protein 5 (LRP5) gene are associated with variation in vertebral bone mass, vertebral bone size, and stature in whites. Am J Hum Genet 2004;74:866-75.

18. Xia W, Hu J, Liu F, et al. New role of LRP5, associated with nonsyndromic autosomal-recessive hereditary hearing loss. Hum Mutat 2017;38:1421-31.

19. Rabbani SA, Arakelian A, Farookhi R. LRP5 knockdown: effect on prostate cancer invasion growth and skeletal metastasis in vitro and in vivo. Cancer Med 2013;2:625-35.

20. Maubant S, Tahtouh T, Brisson A, et al. LRP5 regulates the expression of STK40, a new potential target in triplenegative breast cancers. Oncotarget 2018;9:22586-604.

21. Zhang Y, Xia F, Liu X, et al. JAM3 maintains leukemiainitiating cell self-renewal through LRP5/AKT/betacatenin/CCND1 signaling. J Clin Invest 2018;128:1737-51.

22. .Ayinde $O$, Wang Z, Griffin M. Tissue transglutaminase induces Epithelial-Mesenchymal-Transition and the acquisition of stem cell like characteristics in colorectal cancer cells. Oncotarget 2017;8:20025-41.

23. Wang H, Deng G, Ai M, et al. Hsp90ab1 stabilizes LRP5 to promote epithelial-mesenchymal transition via activating of AKT and $W n t / \beta$-catenin signaling pathways in gastric cancer progression. Oncogene 2019;38:1489-507.

24. Shi X, Zhu M, Kang Y, et al. Wnt/beta-catenin signaling pathway is involved in regulating the migration by an effective natural compound brucine in LoVo cells. Phytomedicine 2018;46:85-92.

25. Dai B, Ma Y, Yang T, et al. Synergistic effect of berberine and HMQ1611 impairs cell proliferation and migration by regulating Wnt signaling pathway in hepatocellular carcinoma. Phytother Res 2019;33:745-55.

26. van Meerloo J, Kaspers GJ, Cloos J. Cell sensitivity assays: the MTT assay. Methods Mol Biol 2011;731:237-45.

27. Bedoya C, Cardona A, Galeano J, et al. Accurate Regionof-Interest Recovery Improves the Measurement of the Cell Migration Rate in the In Vitro Wound Healing Assay. SLAS Technol 2017;22:626-35.

28. Marshall J. Transwell((R)) invasion assays. Methods Mol Biol 2011;769:97-110.

29. Chazotte B. Labeling cytoskeletal F-actin with rhodamine phalloidin or fluorescein phalloidin for imaging. Cold Spring Harb Protoc 2010;2010:pdb.prot4947.

30. Olson MF, Sahai E. The actin cytoskeleton in cancer cell motility. Clin Exp Metastasis 2009;26:273-87.

31. Alblazi KM, Siar CH. Cellular protrusions--lamellipodia, filopodia, invadopodia and podosomes--and their roles in progression of orofacial tumours: current understanding. Asian Pac J Cancer Prev 2015;16:2187-91.

32. Nieto MA, Huang RY, Jackson RA, et al. EMT: 2016. Cell 2016;166:21-45.

33. Wozney JL, Antonarakis ES. Growth factor and signaling pathways and their relevance to prostate cancer therapeutics. Cancer Metastasis Rev 2014;33:581-94.

34. Mabuchi S, Kuroda H, Takahashi R, et al. The PI3K/ AKT/mTOR pathway as a therapeutic target in ovarian cancer. Gynecol Oncol 2015;137:173-9.

35. Burotto M, Chiou VL, Lee JM, et al. The MAPK pathway across different malignancies: a new perspective. Cancer 2014;120:3446-56.

36. Christian F, Smith EL, Carmody RJ. The Regulation of NF-kappaB Subunits by Phosphorylation. Cells 2016;5:12.

37. Wodarz A, Nusse R. Mechanisms of Wnt signaling in development. Annu Rev Cell Dev Biol 1998;14:59-88.

38. Clevers H, Nusse R. Wnt/beta-catenin signaling and disease. Cell 2012;149:1192-205.

39. Hernandez-Maqueda JG, Luna-Ulloa LB, Santoyo-Ramos $\mathrm{P}$, et al. Protein kinase $\mathrm{C}$ delta negatively modulates canonical Wnt pathway and cell proliferation in colon tumor cell lines. PLoS One 2013;8:e58540.

40. Zi X, Guo Y, Simoneau AR, et al. Expression of Frzb/ secreted Frizzled-related protein 3, a secreted Wnt antagonist, in human androgen-independent prostate cancer PC-3 cells suppresses tumor growth and cellular invasiveness. Cancer Res 2005;65:9762-70.

41. Guo Y, Xie J, Rubin E, et al. Frzb, a secreted Wnt antagonist, decreases growth and invasiveness of 
fibrosarcoma cells associated with inhibition of Met signaling. Cancer Res 2008;68:3350-60.

42. Hazan RB, Qiao R, Keren R, et al. Cadherin switch in

Cite this article as: Hong $\mathrm{J}$, Xie Z, Yang Z, Yang F, Liao $\mathrm{H}$, Rao S, Huang X. Inactivation of Wnt-LRP5 signaling suppresses the proliferation and migration of ovarian cancer cells. Transl Cancer Res 2021;10(5):2277-2285. doi: 10.21037/tcr20-3462 tumor progression. Ann N Y Acad Sci 2004;1014:155-63.

43. Kang Y, Massague J. Epithelial-mesenchymal transitions: twist in development and metastasis. Cell 2004;118:277-9. 


\section{Increased expression of LRP5 in metastatic tumor cells}

We detected the background expression of LRP5 protein in different cell lines by Western blot (Figure S1). LRP5 had little expression in colorectal cancer cell line SW480, but highly expressed in its high metastatic cell line SW620. Similarly, LRP5 barely expressed in ovarian cancer cell line HO8910, but highly expressed in its high metastatic cell line HO8910PM, all of which suggest that the expression of LRP5 may be related to cell migration.

\section{Construction and verification of pLVX-IRES-ZsGreen1-DN-LRP5 and pLVX-puro-DN-LRP5 and verification of HO8910PM/DN-LRP5}

To study the functions of LRP5 receptor protein and other related proteins in ovarian cancer cells both in vitro and in vivo, we shall knock down the expression of LRP5 by using dominant negative vector of LRP5. Accordingly, pLVX-puro-DN-LRP5 was constructed to obtain the LRP5 low expression cell line, while pLVX-IRES-ZsGreen1-DN-LRP5 was constructed to detect lentivirals infection efficiency.

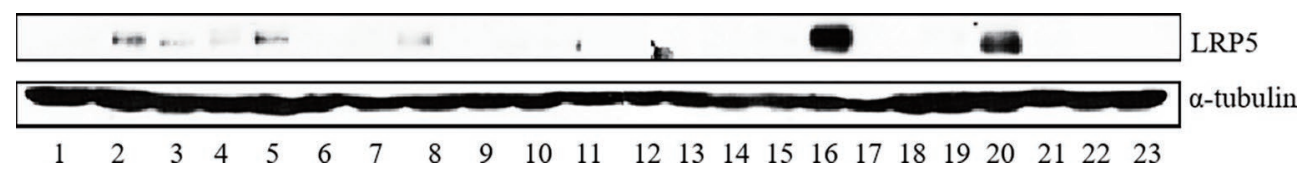

Figure S1 Increased expression of LRP5 in metastatic tumor cells. Human normal liver cells: 1: L02, 2: Chang's liver. Hepatocarcinoma cells: 3: HepG2, 4: HepG2.2.15, 5: Hep3B, 6: 7721, 7: PLC, 8: Huh7, 9: 97-H, 10: LM3, 11: FOCUS, 12: SNU398, 13: Sk-Hep-1. Colon carcinoma cells: 14: LoVo, 15: HCT116, 16: SW480, 17: SW620. OCa cells: 18: A2780, 19: HO8910, 20: HO8910PM, 21: SKOV3. Lung cancer cells: 22: H460, 23: H446.

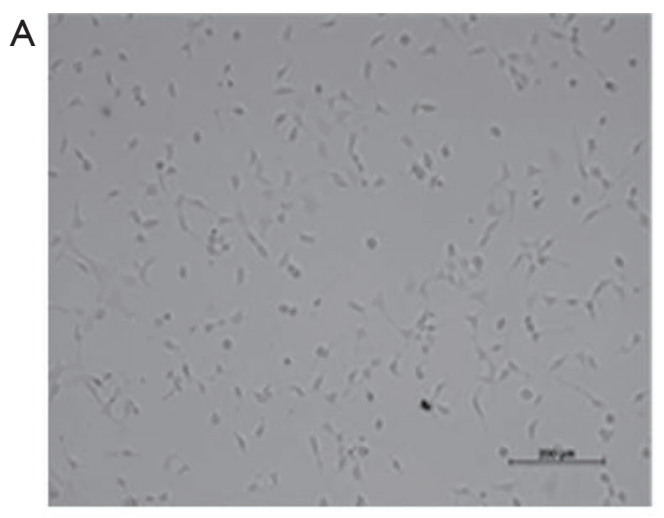

C

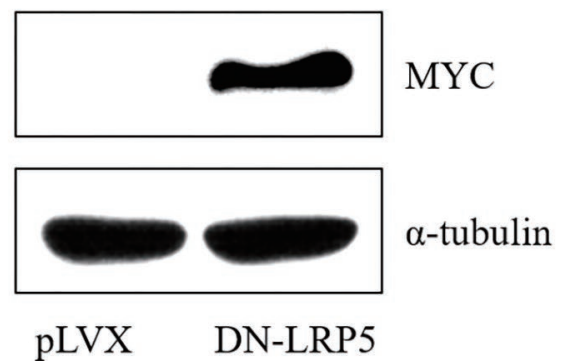

B

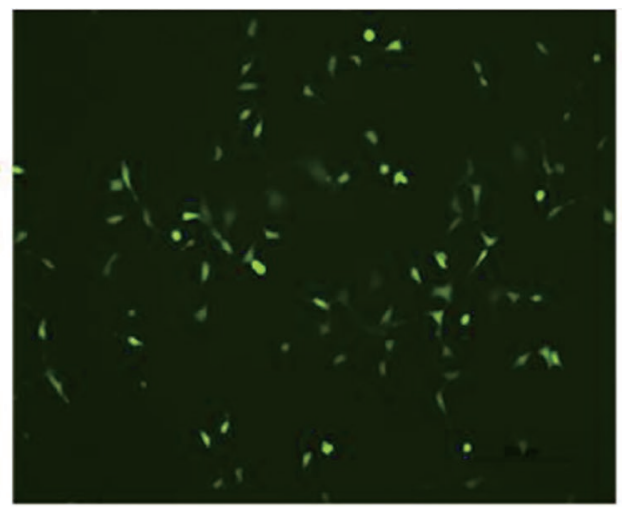

D

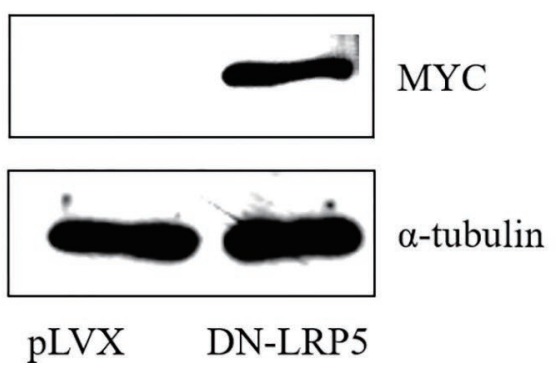

Figure S2 Verification of HO8910PM/DN-LRP5. (A) Light microscopy observation of HEK293T infected with pLVX-IRES-ZsGreen1DN-LRP5 virals (×100); (B) fluorescence microscopy observation of HEK293T infected with pLVX-IRES-ZsGreen1-DN-LRP5 virals $(\times 100)$; (C) Western blot analysis for expression of DN-LRP5 (myc) in pLVX-puro-DN-LRP5 virals in HEK293T; (D) Western blot analysis for expression of DN-LRP5(myc) in pLVX-puro-DN-LRP5 virals in HO8910PM. DN-LRP5, dominant negative plasmid of LRP5. 
To obtain packaged recombinant lentivirus, HEK 293T cells were co-transfected with recombinant lentivirus expression plasmids and lentivirus packaging plasmids psPAX2 and pMD2.G. After infection, expression of a large number of green fluorescent proteins in HEK 293 T cells was observed by fluorescence microscopy (Figure S2A,B), while expression of DNLRP5 protein was detected by Western blot (Figure S2C).

To obtain the cell line stably expressing DN-LRP5, HO8910PM cells were infected with the recombinant lentivirus and resistant cell clones were selected with puromycin. Consistently, the expression of DN-LRP5 in positive clones was detected by Western blot (Figure S2D).

\section{DN-LRP5 regulates EMT and multiple cancer-associated pathways}

To see if DN-LRP5 are associated with EMT and some cancer-related pathways, multiple EMT or cancer-associated proteins are checked by Western blotting (Figure 3), and the quantitative densitograms of Figure 3 are shown in Figure S3.
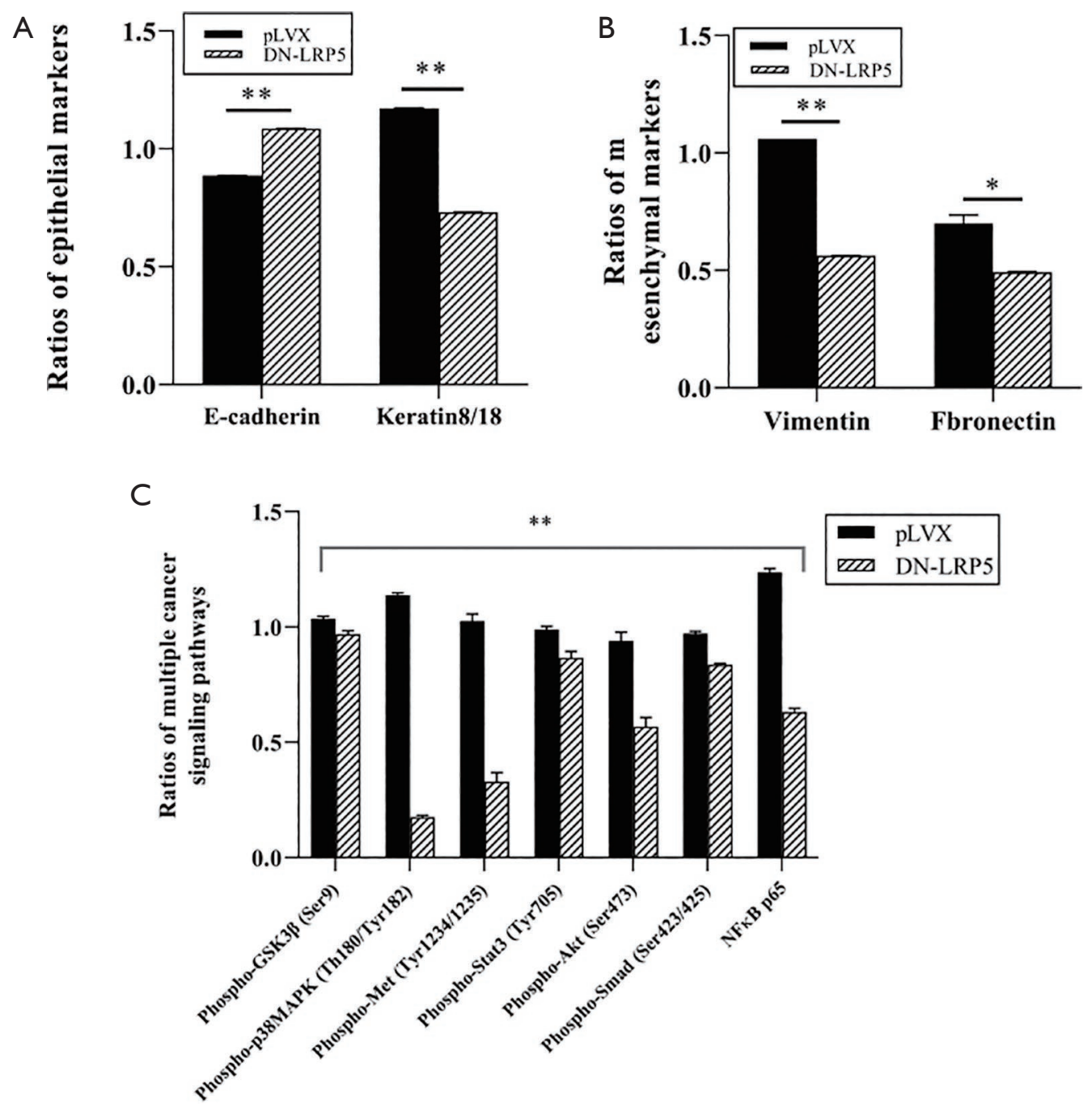

Figure S3 Western blotting densitograms of DN-LRP5 associated EMT and multiple cancer-associated pathways. (A) Ratios of epithelial markers verse tubilin; (B) ratios of mesenchymal markers verse tubilin; (C) ratios of multiple cancer signaling pathway proteins verse tubilin. DN-LRP5, dominant negative plasmid of LRP5; EMT, epithelial-mesenchymal transition. 